\title{
The Impact of Community Opening on Road Access
}

\author{
Qingya Zhou \\ School of Control and Computer Engineering, North China Electric Power University \\ Beijing, China
}

Keyword:Communityopening, Thenumber of optimal paths, The average delays of crossing,The traffic capacity

\begin{abstract}
The communityopening has attracted wide attention and discussion. In this paper, two problemsare solved.One is to select appropriate evaluation indexesanddo quantitativelyanalyses for each index. And another one is to establish a traffic index model. For the first problem, three kinds of indexes are chosen to be as the evaluation indexes. They are the number of optimal paths, the average delaysof crossing and the traffic capacity. Through calculatingthenumber of optimal pathsbetween perfect point grids, establishing a delay model, and establishing the traffic capacity model, the impact of community opening on road access can be discussed. It can be concluded that if the number of optimal paths becomes larger, the average delays of crossing becomes smaller and the road capacity becomes larger,the traffic on the surrounding roads will be better. For the second problem, themathematical model which is called traffic index is established. It is based on fuzzy comprehensive evaluation method. Firstly, to define an evaluation set which is flarger, large, medium, small, smaller $\}$. Then, to define a factor set which is $\{$ the number of optimal paths, the average delays of crossing, the traffic capacity\}. Next, to determine the weight of each factor based on analytic hierarchy process. The set of the weight can be calculated which is $(0.8058,-0.1325$, 0.326). Lastly, to determine the fuzzy judgment matrix.The community opening on road access can be quantified based on the traffic index. However, a new more accurate and effective model is supposed to be found when the surrounding environment, roads and public facilitiesmake certain changes.
\end{abstract}

\section{Introduction}

The communityopening has attracted wide attention and discussion.Its impact on the surrounding road traffic brings both advantages and disadvantages, which cannot be generalized. The impact of the community opening on surrounding roads is discussed in this paper.A quantitative basis for scientific decision-making is provided, which helps to design a community.

\section{Model hypothesis and symbolic agreement}

Suppose that the structure of road, the traffic accident rate and other environmental factors change little during the studying.

Set the symbolic agreement as [Table 1].

Table 1 symbolic agreement

\begin{tabular}{cc}
\hline variable & symbol \\
\hline road traffic index & $\mathrm{W}$ \\
the number of optimal paths & $\mathrm{A}$ \\
the average delaysof crossing & $\mathrm{B}$ \\
the traffic capacity & $\mathrm{C}$ \\
road accessibility & $\mathrm{R}$ \\
road status vector & $\overrightarrow{\mathrm{x}}$ \\
\hline
\end{tabular}




\section{Solution of the Number of Optimal Paths}

The trafficgrid network has a special structural feature. Shown as Fig.1, multiple nodes are located on the same straight line. If the two nodes are not on the same straight line, there will be multiple optimal paths between the pair of points. The question to be discussed is the optimal number of paths between grid points. The passing time of each edge is supposed to beone, and the number of optimal paths from $\mathrm{v}_{00}$ to $\mathrm{v}_{\mathrm{nm}}$ is the solution which is supposed to be found. $\mathrm{G}$ refers to a given plane non-directional grid traffic network. The set $V$ is $\left\{\mathrm{v}_{\mathrm{ij}} ; \mathrm{i}=0,1,2, \cdots ; \mathrm{m}, \mathrm{j}=0,1,2, \cdots ; \mathrm{n}\right\}$. Based on mathematicalinduction, it can be concludedthat:

$\mathrm{A}=\mathrm{N}(\mathrm{vnm}, \mathrm{v} 00)=\sum_{i=1}^{m} N(\mathrm{vn}-1, \mathrm{i}, \mathrm{v} 00)+1=\frac{(\mathrm{m}+1)(\mathrm{m}+2)(\mathrm{m}+3) \cdots(\mathrm{m}+\mathrm{n}-1)}{\mathrm{n} !}=\frac{(\mathrm{m}+\mathrm{n}) !}{\mathrm{m} ! \mathrm{n} !}$ “ 1 ”

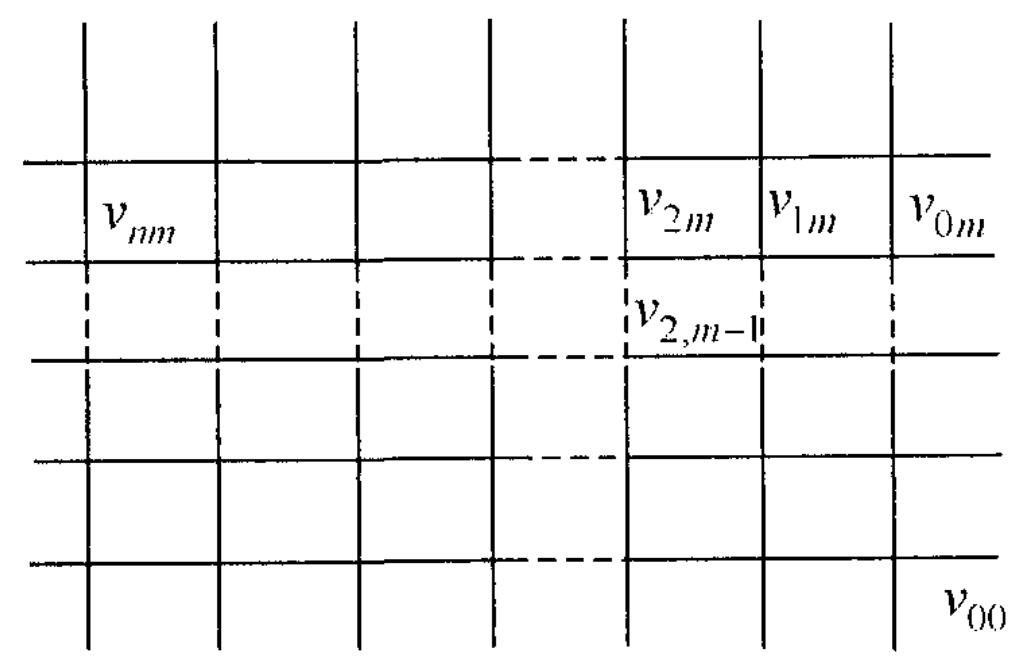

Fig. 1

With the solutionsabove, the optimal number of paths between any point pairs on the gridcan be found. For the node of column $n+1$ and line $m+1$, suppose that the grid on the road traffic network, on which each side of the passage of time is one,then,its number of optimal paths of $\mathrm{v}_{00}$ to $\mathrm{v}_{\mathrm{nm}}$ is $\frac{(m+n) !}{m ! n !}$. And the larger the number of optimal paths is, the better the traffic on the surrounding roads will be.

\section{Establishment of the Delay Model}

Formula for the calculation of average delaysof crossingis:

$$
\mathrm{B}=\frac{0.5 \mathrm{~T}\left(1-\frac{\mathrm{tg}}{\mathrm{T}}\right)}{1-\left[\min (1, \mathrm{x}) \cdot \frac{\mathrm{tg}}{\mathrm{T}}\right]} “ 2 ”
$$

T refers to signal cycling length,Tgrefers tothe effective time of green light,and $\mathrm{x}$ refers totraffic saturation.

It can be concluded that the greater the average delays ofcrossing is, the worse the traffic of the surrounding roads will be.[1] 


\section{Establishment of the Traffic Capacity Model}

The communityopeningin large cities will inevitably influence theperformance of the surrounding road network, which makes changes tothe overall performance of the urban road network. In order to analyze the changesto the performance of urban local network, the reliability-evaluation index of road networkis established which is called the traffic capacity model.

Assuming that the local road network has n sectionsin total. The internal road sections of the cell are included as well. And the reliability of the link is known.The xi (0 or 1) of link iis defined as:[2]

$$
x_{i}=\left\{\begin{array}{lr}
1 & \text { community opening } \\
0 & \text { community closed }
\end{array}\right.
$$

If the vector is the "state vector" of the road network, the service state $\varphi$ between the network nodes can be defined as:

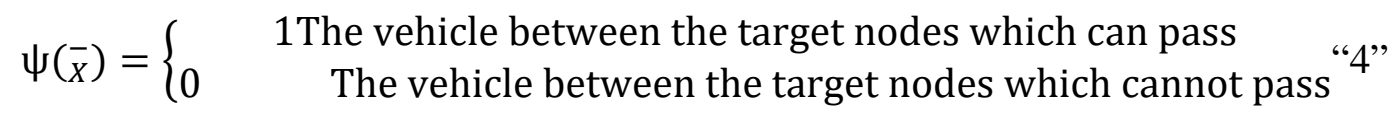

The reliability of the road section is expressed based on the expected value of the probability-variable. The reliability of the road network is given based on the expected value of the system constructor. The form of the constructor is determined by the road network as:

$$
\begin{aligned}
& r_{i}=E\left(x_{i}\right) “ 5 ” \\
& \mathrm{R}=\mathrm{E}[\psi(\bar{x})] “ 6 ”
\end{aligned}
$$

It can be concluded that the greater the road capacity is, the better the traffic on the surrounding roads will be.

\section{Establishment of Traffic Indexes Model}

Themathematical model called traffic index is established based onfuzzy comprehensive evaluation method [3]. Firstly, to define an evaluation set which is \{larger, large, medium, small, smaller\}. Then, to define a factor set which is the number of optimal paths, the average delays of crossing, the traffic capacity\}. Next, to determine the weight of each factor based on analytic hierarchy process. The set of the weight can be calculated which is $(0.8058,-0.1325,0.326)$. Lastly, to determine the fuzzy judgment matrix.The community opening on road access can be quantified based on the traffic index.

The traffic road index Wis determined as the target layer. The number of optimal paths $\mathrm{A}$, the average delays of crossing $\mathrm{B}$, and the traffic capacity Care determined as the criterion layer.Firstly, the main quantitative factors are quantitatively processed.And the quantification value of the factors is given. The evaluation set \{larger, large, medium, small, smaller refers to the value setwhich is $\{5,4,3,2,1\}$. Here the Cauchy distribution membership functionis given.

$$
\begin{aligned}
& \mathrm{f}(\mathrm{x})= \begin{cases}{\left[1+a(x-b)^{-2}\right]^{-1},} & 1 \leq x \leq 3 \\
\operatorname{cln} x+d, & 3 \leq x \leq 5\end{cases} \\
& \mathrm{f}(5)=1, \mathrm{f}(3)=0.8, \mathrm{a}=1.1086, \mathrm{~b}=0.8942, \mathrm{c}=0.3915, \mathrm{~d}=0.3699 .
\end{aligned}
$$

Then the membership function can be:

$$
\mathrm{f}(\mathrm{x})= \begin{cases}{\left[1+1.1086(x-0.8942)^{-2}\right]^{-1},} & 1 \leq x \leq 3 \\ 0.3915 \ln x+0.3699, & 3 \leq x \leq 5\end{cases}
$$




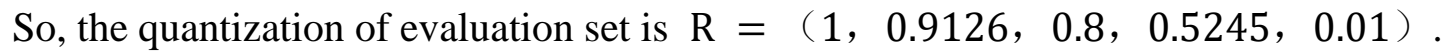

To determine the weight of each factor based onanalytic hierarchy process.Build each judgment matrix according to the indexes.

Table 2 judgment matrix

\begin{tabular}{cccc}
\hline W & A & B & C \\
\hline A & 1 & -5 & 3 \\
B & $-1 / 5$ & 1 & $-1 / 3$ \\
C & $1 / 3$ & -3 & 1 \\
\hline
\end{tabular}

Using the software MATLAB to calculate the weight $\alpha, \beta$, and $\gamma$.And $\alpha$ refers to the number of optimal paths, $\beta$ refers tothe average delays of crossing, $\gamma$ refers to the traffic capacity. Then the weight vector can be got.

$$
A=(\alpha, \beta, \gamma)=(0.8058, \quad-0.1325, \quad 0.326) “ 9 ”
$$

To do consistency-check. $\lambda \max =3.039$. The consistency criterion CI is determined.CI $=$

$$
\frac{\lambda \max -n_{\text {“ }}}{\mathrm{n}-1} 10 \text { ” }
$$

Solutions can be calculated.

$$
\begin{aligned}
& \mathrm{CI}=\frac{\lambda \max -\mathrm{n}}{\mathrm{n}-1}=0.0195 \\
& \mathrm{CR}=\frac{\mathrm{CI}}{\mathrm{RI}}=0.037<0.1
\end{aligned}
$$

It can be concluded that it satisfies the consistency.So, thetraffic index $\mathrm{W}$ can be got by multiplying each index.

$$
\begin{gathered}
\mathrm{W}=\alpha \times \frac{(m+n) !}{m ! n !}+\beta \times \frac{0.5 T\left(1-\frac{t_{g}}{T}\right)}{1-\left[\min (1, x) \times \frac{t_{g}}{T}\right.}+\gamma \times \mathrm{E}[\psi(\bar{x})] \\
=0.8058 \times \frac{(m+n) !}{m ! n !}-0.1325 \times \frac{0.5 T\left(1-\frac{t_{g}}{T}\right)}{1-\left[\min (1, x) \times \frac{t_{g}}{T}\right.}+0.326 \times \mathrm{E}[\psi(\bar{x})]
\end{gathered}
$$

\section{Conclusion}

We can get that $\mathrm{R}=(1,0.9126,0.8,0.5245,0.01)$.

If $\mathrm{W} \geq 1$ or $1-\mathrm{W} \geq \mathrm{W}-0.9126$, which is $\mathrm{W} \geq 0.9563$, it refers to community opening has a very perfect impact on road access;

If $0.9162-\mathrm{W} \geq \mathrm{W}-0.8$, which is $0.9563 \geq \mathrm{W} \geq 0.856$, it refers to community opening has a good impact on road access;

If $0.8-\mathrm{W} \geq \mathrm{W}-0.5245$, which is $0.8561 \geq \mathrm{W} \geq 0.66225$, it refers to community opening has a not bad impact on road access;

If $0.5245-\mathrm{W} \geq \mathrm{W}-0.01$, which is $0.66225 \geq \mathrm{W} \geq 0.26225$, it refers to community opening has a bad impact on road access;

If $0.26225 \geq \mathrm{W}$, it refers to community opening does harm to road access.

It can be concluded that if the number of optimal paths becomes larger, the average delays of 
crossing becomes smaller and the road capacity becomes larger,the traffic on the surrounding roadswill be better.However, a new more accurate and effective model is supposed to be found when the surrounding environment, roads and public facilitiesmake certain changes.

\section{References}

[1] Zhuang Yan, ZengWenjia. Research on Delay Calculation Model of Signalized Intersection [J] .Journal of Shenzhen University (Science \& Technology Edition), 2006,23 (4): 309-313.DOI: 10.3969 / j.issn.1000-2618.2006. 04.005.

[2] Information on

http://wenku.baidu.com/link?url=gokSYab0v61FxnQxo0JMHcW3DSZl7nCBHOxZH8_iB-9mtAn 9H7PwQ5TVvp9oUv5Kb8Iz6_TBLwc6h_seq60E07nIq5Bzh2IP3vR5vr--qFS. 2016/09/11

[3] Secretary Shoukui, Sun Zhaoliang. "Mathematical modeling algorithm and application." Defense Industry Press .2016 January 